ANADOLU UNIVERSITY JOURNAL OF EDUCATION FACULTY

\title{
Revisiting Social Construction of Knowledge in Asynchronous Computer Mediated Communications (CMC): What to Change*
}

\author{
Çiğdem Suzan ÇARDAK ${ }^{1}$
}

Article Type: Research Article

Application Date: 12.01.2020

Accepted Date: 30.01 .2020

To Cite This Article: Çardak, Ç. S. (2020). Revisiting Social Construction of Knowledge in Asynchronous Computer Mediated Communications (CMC): What to Change. Anadolu University Journal of Education Faculty (AUJEF), 4(1), 67-87.

\begin{abstract}
This study focuses on social construction of knowledge in asynchronous online forum discussions. Social construction of knowledge and how to analyze the quality of interaction during computer mediated communications (CMC) were studied for decades. Interaction Analysis Model (IAM) is one of the mostly used models for determining the quality of CMC. The author also used IAM while analyzing the content of CMC activities in the past. In this study, the same analysis of $\mathrm{CMC}$ was conducted again with different participants and discussion topics in order to see what changes in the current study if the voluntary/mandatory participation to discussions, prior interpersonal familiarity, moderating behavior during the $\mathrm{CMC}$, and discussion technique change, comparing to previous study of the author. The aim of this paper is to identify the levels of social construction of knowledge in CMC of the postgraduate students of a university in Turkey. The data of this case study is composed of CMC messages and views of the participants written on an anonymous open-ended questionnaire. Quantitative content analysis and thematic analysis were conducted on the data set. The analysis of the messages by IAM of this case study gave slightly better results than the previous study of the author, and some suggestions were put forward for the future research.
\end{abstract}

Keywords: Social construction of knowledge; computer-mediated communications (CMC); online discussions; interaction analysis model (IAM)

\footnotetext{
${ }^{*}$ This study was presented as oral presentation at the $11^{\text {th }}$ International Conference on Educational Technology and Computers (ICETC 2019), Amsterdam, Netherlands, October 28-31, 2019.

${ }^{1}$ Assist.Prof.Dr., Anadolu University, csbelikusakli@anadolu.edu.tr, ORCID: https://orcid.org/0000-0002-16431599 (Corresponding author)
} 


\section{INTRODUCTION}

Interaction is considered as one of the key factors of learning both for face to face and e-learning experiences. Interaction in learning process has such an importance that learners construct knowledge during their interactions in the learning environment ( $\mathrm{Su}$ et. al, 2005). Gómez \& Hernández (2018) give an analogy of patchwork quilt while defining interaction as "the collection of unique messages sewn together, resulting in socially constructed knowledge" (p. 285). Previous studies also supports the contributions of interaction into e-learning process (eg. Arbaugh \& Benbunan-Fish, 2007; Çardak, 2016a; LaPointe \& Gunawardena, 2004; Rovai \& Barnum, 2003; Sher, 2009). Moore (1989) defines three types of interaction for distance learners: learner-learner, learner-instructor, learner-content interaction. All three interaction types have crucial importance on constructing new knowledge during the learning process.

Social interactions, interactions of the learners with other individuals in the learning environment, are invaluable for e-learning processes, especially when curriculum is based on social constructivism. Instructors can design dozens of learning activities that accommodate learners' social interactions. Discussion activities conducted in the conventional and e-learning processes in line with the instructional aims stated in the course program is one of the functional ways for enhancing social interactions and helping learners think deeply into the content. Debates, panels, small and large group discussions, inner circle/outer circle are some of the widely used discussion techniques in conventional learning processes for all education levels, which are also conducted in e-learning environments. Various synchronous and asynchronous computer mediated communication (CMC) systems are used as a medium of such discussion activities, which enhance social interactions.

Computer-Mediated Communication (CMC) is "growing rapidly as a mechanism for human communication and interaction" (Lin \& Huff, 1988, p.1). Initial use of CMC was seen as a faster form of letters (Savignon \& Roithmeier, 2004), then parallel to the developments in Internet technologies, CMC systems evolved to voice mail, videoconferencing, asynchronous forum and bulletin board, sychronous chat tools and features of social networking sites today. Whilst CMC systems have virtues such as low cost, asynchronicity, and efficiency, main limitation is their lack of human contact (Lin \& Huff, 1988). In face-to-face settings, "facial expressions, direction of gaze, posture, dress, non-verbal, and vocal cues" are contributing factors to social presence (Tu, 2000, p. 28). The absence of these factors in $\mathrm{CMC}$ environments might hinder the participants from building social presence and thus a learning community, whilst social presence is seen as a critical influence on learners' social interaction in elearning environments $(\mathrm{Tu}, 2002)$. Social presence is defined as "the degree of feeling, perception, and reaction to another intellectual entity in the CMC environment" (Tu \& McIsaac, 2002, p. 146). In order to enhance social interactions and effectiveness of discussions through CMC, social presence should be felt by each member of the learning community.

The study of Gunawardena and Zittle (1997) showed the social presence as a strong predictor of satisfaction in a computer conference. Accordingly, they advise designing techniques deliberately to enhance social presence. Motivating learners actively participate in $\mathrm{CMC}$ activities is a need for building social presence. To motivate the learners participate actively in CMC sessions, most of the e-educators make the $\mathrm{CMC}$ activities one of the mandatory components of the course programs in higher education and the participation into this activities are graded. Nevertheless, grading the participation in quantity does not guarantee the social presence, social interaction and higher levels of learning during CMC. Assessing the quality of the CMC activities requires analyzing the content in various aspects in which the instructional aims of the related curriculum implies. For instance, if the learning theory basis of the 
curriculum is cognitivist-behaviorist, instructional objectives taken into account while examining the quality of the $\mathrm{CMC}$ activities designed and implemented according to the instructional aims. However, the communicative structure of CMC systems call for much more social constructivist activities regarding to social constructivist curricula. In the latter case, the one who would like to assess the quality of the CMC activities might analyze the social interactions in order to understand how knowledge and cognition are collaboratively produced by the participants of the CMC (Zhao, Liang, \& Liu, 2016).

The focus of numerous research on social construction of knowledge is conducting interaction analysis models in order to examine the contents of the asynchronous message transcripts (Lucas, Gunawardena \& Moreira, 2014). One empirically validated interaction analysis model, which focuses on social construction of knowledge in CMC, is Interaction Analysis Model (IAM) of Gunawardena, Lowe and Anderson (1997). Their interaction analysis technique is an initiative to understand the social construction of knowledge within a group of learners in CMC activities. They analyzed the content of debate type CMC sessions and by conducting grounded theory, they developed interaction/content analysis model. This model is based on the principles of social constructivism and its main assumption is "construction of knowledge is the result of interaction, meaning negotiation and building of a shared understanding" (Lucas et al., 2014, p. 576).

IAM consists of five phases and each phase has three to five operations (Gunawardena et al., 1997, p. 414). Phase I is "sharing/comparing of information", and includes five operations: "sharing an observation or opinion; agreements; examples; questions and answers for clarifying; identifying a problem". Phase II is "the discovery and exploration of dissonance and inconsistency among ideas, concepts or statements", and it includes three operations: "stating disagreements; clarifying disagreement through questioning; clarifying participant's positions". Phase III is "negotiation of meaning/coconstruction of knowledge", and includes five operations: "negotiation of the meaning of the terms; arguments; identification of areas of agreement; proposing new statements embodying co-construction; proposing accommodating metaphors or analogies". Phase IV is "testing and modification of proposed synthesis or co-construction", and includes five operations: "testing the new proposed arguments against participants' culture; testing against cognitive schema; testing against personal experience, testing against formal data, testing against literature". Phase V is "applications of newly constructed meaning", and includes three operations: "summarizing the agreements; applications of new knowledge; metacognitive statements by the participants related to their understanding".

There are many studies which conducted interaction analysis on $\mathrm{CMC}$ by using Interaction Analysis Model (IAM) (Heo, Lim \& Kim, 2010; Hew \& Cheung, 2011; Hou, Chang \& Sung, 2008; Gómez, 2018; Lu \& Jeng, 2006; Paulus, 2007; Tan, Chai, \& Hong, 2008) mainly in the context of higher education or post-graduation (Lucas et al., 2014). The author also conducted a naturalistic study as a case on this topic several years ago with the participation of postgraduates and found low levels of social construction of knowledge in CMC according to IAM (Çardak, 2016). Though CMC systems and activities are still important components of e-learning processes, why low levels of social construction of knowledge occur in most of the CMC activities seeks for answer. Asking "what to change" for high level of social construction of knowledge in CMC activities comparing to previous experiences might be more crucial than answering this question. Because the question "why do low levels of social construction of knowledge occur in most of the CMC activities?" is such hard question which requires estimating many factors. According to author of this study, each instructor or e-moderator should ask herself/himself what to change for enhancing social construction of knowledge in CMC activities if her/his previous CMC sessions do need meet the criteria of high level of social construction of 
knowledge. The motivation behind revisiting the level of social construction of knowledge in CMC activities for the author is her curiosity on what changes if four structural differences for the forum discussions are considered comparing to author's previous study (Çardak, 2016), as students' interpersonal familiarity, only-moderating behavior of the instructor/moderator and debate type discussion technique, in the CMC of the current study?

The aim of this study is to conduct asynchronous forum discussions (debate and free-flow discussions) as CMC in Turkish language with the voluntary participation of the postgraduate students of the Curriculum and Instruction MSc and PhD programs, and identify the levels of social construction of knowledge according to IAM in a university in Turkey. Following research questions are answered:

1. What are the views of the participants regarding the discussion sessions?

2. Which phases of social construction of knowledge occur according to IAM in CMC?

The difference between the findings of the current and the previous studies of the author are compared and discussed in order to gain some insights about what changes or does not change regarding $\mathrm{CMC}$ when the previously stated four aspects (voluntary basis, familiarity, moderating behavior, discussion technique) change.

\section{METHOD}

\subsection{Design of the Study}

The study was designed as a case study. The examined case is asynchronous forum discussion sessions in discussion-forum-type CMC system moderated by the author with the participation of postgraduate learners of the Curriculum and Instruction MSc and $\mathrm{PhD}$ programs of a university in Turkey.

In the spring term of 2019, all of the graduate students of the Curriculum and Instruction department were invited to participate in CMC voluntarily with a message posted on the department's Facebook group. Among the 47 graduate students (22 master's, 25 doctorate-level) of the program, only 14 students agreed to participate in the CMC. Because of the final exams and other responsibilities of the participants, the discussions started in June 2019, and after six weeks discussion, the discussions were ended in the late July 2019. During this period, two independent discussion sessions were organized. The first discussion topic was constructed as a debate on a specific topic: "Which one is best for active learning: e-learning vs. face-to-face?" The second discussion topic was constructed as a free flow discussion on a general topic: "Globalization and e-learning".

In order to provide a medium for the CMC, the forum tool of the Blackboard Learning Management System provided by the university was used. Blackboard course named as "Sosyal Yapılandırmacı Çevrimiçi Tartışmalar (Social Constructive Online Discussions)" in Turkish was opened as a stand-alone course only used for the CMC of this study. In other words, CMC of this study was not a component of any course in the programs of Curriculum and Instruction. All of the volunteer participants were registered to the Blackboard by providing their user name and passwords. Therefore, the $\mathrm{CMC}$ were only open to the participants and the moderator of the discussions of this study.

The findings derived from the current content analysis are compared with the previous study of the author (Çardak, 2016). In order to interpret the findings of this case study comparing to previous one, the structural differences of the current and the previous CMC are given on Table 1. 
Table 1. Structural Differences between the Current and the Previous CMC Studies

CMC of the Current Study

Participation to CMC is optional, only for volunteer participants: No grades, and no participant incentives
CMC of the Previous Study (Çardak, 2016)
CMC is a requirement of a compulsory course:

Active participation was graded.
Master's and doctorate-level students of the same major: curriculum and instruction: Most of the participants know each other beforehand.

One debate type discussion, one free flow discussion
Doctorate-level students of various programs of various institutes: Most of the participant do not know each other beforehand.

No debate type discussion, whole free-flow discussions

The author of both studies moderated the CMC and the similar asynchronous forum tool of the learning management systems provided by the university was used as a medium of the discussions. Participants of the previous and the current study and the discussed topics are different in two studies. Moreover, as showed on Table 1, there are four main structural differences between the CMC of the current and the previous study. The first difference is participating into CMC being voluntary or compulsory. The participants of the previous study should have to actively involve in the discussions because CMC was mandatory and graded. In the present case, participating into discussions was voluntary and the $\mathrm{CMC}$ was not a component of any course program. The second structural difference is familiarity of the participants beforehand. Most of the participants of the previous case study were not know each other before the start of the discussions. They were enrolled in the course from various doctorate-level programs of various institutes. On the other hand, most of the participants of the current study had already known each other and they were somehow familiar with other participants and the moderator of the discussions. The third structural difference is the discussion technique conducted during CMC. During the CMC sessions of the previous study, 11 free-flow discussions were conducted for each of the 11 units of the course. In this study, two discussion sessions were conducted: one as a debate and the other one as a free-flow as the previous study's CMC. The final structural difference is the moderating behaviors of the moderator. In the case of the previous study, the moderator was also the co-participant of the discussion session. In the current case, the moderator refrained herself from sharing messages just like the participants, instead she moderated the flow of the discussions.

\subsection{Participants}

The participants of the current study are the master's and doctorate-level students of the "Curriculum and Instruction" program of the Graduate School of Educational Sciences at one of the leading universities in Turkey. 14 volunteer graduate students were willing to participate, but not all were active during six weeks. 10 participants actively participated in the Discussion 1, while eight participants participated in Discussion 2. If two discussions are considered together, 10 participants actively involved in the discussions while four of the volunteers did not sent a message. Three of ten participants were 
registered to master's program of the Curriculum and Instruction and six of them to doctorate program of the same major. One of the participants graduated from the doctorate-level program few years ago and voluntarily participated in the discussions.

Participants were familiar to each other from the postgraduate level courses and/or seminar presentations at the end of each semester before the CMC of this study. Indeed, they are in the same social networks of the program. Thus, they previously had a sense of community in the same program of the same graduate school.

\subsection{Researcher as the Moderator of the Discussions}

The author has 16 years-experience on e-moderating and interested in effectiveness of teachinglearning process of online, blended and face-to-face courses. She conducted various studies and wrote a $\mathrm{PhD}$ thesis on interaction in a blended course. The author, as a researcher, conducted content analysis in one of the CMC of the online courses in the past by using IAM. In the previous study of the researcher on IAM, she moderated a whole term forum discussions of a postgraduate level online course, which was a compulsory course for the doctorate level students of various programs in Turkey. The reason of revisiting the IAM again is the author's suspicion about what if the some aspects of the forum discussions change, the results regarding the levels of social construction of knowledge might change or not. The author of this study conducted, moderated and analyzed the interactions in CMC by using IAM in the current study. Researchers who conducted this analysis before should know well that the content analysis of CMC with IAM is a very compelling task, which might require special interest to theory of social constructivism, comprehending the flow of the whole discussion session and somehow experience on coding according to IAM because it is much more than just counting the recurring words or sentences. Thanks to the author's previous case study, she gained experience while coding 345 messages of the whole term CMC and conducting reliability studies with independent researchers (Çardak, 2016).

\subsection{Data Collection and Analysis}

Two types of data are collected in the current CMC study: CMC records and open-ended electronic questionnaire.

\subsubsection{Records of Discussion Forum of Blackboard}

Blackboard discussion forum records gave 62 messages in total for both discussions. 33 messages were sent to Discussion 1, and 29 message to Discussion 2. During Discussion 1, moderator posted 5 messages, whereas the participants sent 28 messages. During the Discussion 2, moderator shared 11 messages while the participants sent 18 messages. Because the first direction is debate on a specific topic, moderator did not need to intervene the flow of the discussion as much as the second discussion. Second discussion was a free-flow discussion on a wider topic and thus the moderator occasionally posted messages to remind the topic, and summarize the views. At total, 46 messages posted by the postgraduate students to both discussion forums were analyzed. The content of the moderator's messages was not analyzed because she was not the co-participant of the discussions. However, in the previous study of the author, the content of the moderator's messages analyzed as well as the participants' messages, because the moderator was also participant of the discussions. 
In the current study, discussion messages were analyzed through quantitative content analysis by using IAM. A single message was taken as a "unit of analysis", and in most of the messages, evidences of more than one phases were found and coded into related operations of the phases. The analysis process of the CMC messages was occurred as the following:

- All of the messages were read before starting analysis in order to comprehend in deep the flow of the discussion.

- A specific code was assigned manually to each message. For example; D2(5) refers to fifth message of the Discussion 2.

- Each message was read more than three times, considering the flow of the discussion; then, a code was assigned manually to each separate evidence of phases in the message. For instance: D1(2)P1(A) refers to an evidence of Operation A of Phase 1 in the second message of the Discussion 1.

- Reliability study: An independent researcher was informed about the phases and operations of IAM, and asked for checking the codings of the researcher according to IAM on sample data. Apart from few codings, independent researcher confirmed and agreed upon all the codings assigned by the researcher. The controversial codings were discussed until arriving at a consensus by considering the flow of the discussions and the IAM.

- Codes in the messages were written in related cells on the coding sheet of IAM, which shows each Phase and operations.

- Total numbers of codings and ratios in each operations of the phases were calculated and presented in tables with same representative examples as evidences of various operations.

\subsubsection{Open-ended questionnaire}

Participants were asked to answer an anonymous questionnaire at the end of the CMC. The openended questions were written by the researcher according to design of the CMC in the current study, expert views, and interview questions of the previous study, because half of the questions were taken from the interview form, developed according to experts' view for the previous study. The questionnaire consisted of eight open-ended questions, which ask the views of the volunteer participants regarding their extent of participation in discussions, reasons of participating or not participating, both discussion topics, process and outcomes of each discussion, and other views and suggestions. The questionnaire was delivered as an electronic survey and the related link was shared with the participants. Among the 14 volunteer participants, nine of them wrote on the questionnaire form.

Thematic analysis was conducted on the qualitative data collected through open-ended questionnaire:

- All of the answered questionnaires were downloaded from the electronic source and uploaded to NVivo12, each as a separate .docx document.

- After reading the nine documents in detail, main categories were created regarding the questions on the questionnaire.

- Each data source was read in detail and sub-categories emerged gradually. Researcher prepared an initial list of themes and sub-themes. 
- Reliability study: Three of the nine questionnaires were read in detail from an independent researcher and each meaning in the sentences grouped into 41 categories manually by the independent researcher on her own. Then the author conducted a meeting with the independent researcher. Two researchers compared 41 codings of the sample data with the initial codings of the researcher and found that 34 categories of the independed researcher are represented in the initial list of sub-themes, and 7 categories are not found in the initial list. Thus, the initial agreement ratio between the researchers are found to be $83 \%$ according to interrater reliability formula (number or agreements/ (number of agreements + disagreements) x100). Two researchers discussed on each of the seven contradictory categories respecting the data and arrived at a consensus. Accordingly, the author conducted required revisions in the list of the sub-themes.

- Related direct quotation examples of the sub-themes were presented by indicating the questionnaire code as Q1, ..Q9.

\section{FINDINGS}

Findings are presented according to two reseach questions. Firstly, the participants' views regarding the discussions are given, and then the findings of the content analysis of the CMC transcripts are reported.

\subsection{Views of the Participants Regarding the Discussions}

Views of the participants are classified under five main themes:

Participation

> First discussion: «E-learning or face to face? Which one is better for active learning?»

> Second discussion: «Globalization and e-learning»

$>$ General views about the whole CMC process

$>$ Suggestions

\subsubsection{Participation}

Participants' views about their participation in both discussions are classified into the following sub-themes:

- Being active in both discussions

- Being more active in the first discussion

- Responding 3-4 times

- Not participating actively enough

Postgraduate student stated participating both discussion sessions, or being more active in the first discussion. They also stated responding the other participants' messages three to four times or not participating actively enough. 
"I tried to participate in both discussions and state my views whenever I found time" Q3

"Especially, I participated into the first discussion more than the second one." Q4

Moreover, participants' reasons for voluntarily participating in CMC of this study are questioned. According to their views, curiosity of the CMC, sharing views, brainstorming, learning new things, observing the moderator's style, experiencing a different learning process and easy and known discussion topics are their reasons for participating voluntarily. One of the participants explained his/her views as the following:

"The aim of my participation was to share my own views and read the others.." Q5

In addition to reasons for participating, postgraduate students' obstacles to participating actively enough are limited spare time in their life, facing technical problems of connecting from smart phones, summer holiday time, not carrying laptops with himself/herself. One of the participants stated her/his views as follows:

"I could not continue the discussion because of personal and technical reasons. The study came across to my heavy lead on work and personal life. Indeed, the mobile interface of the system did not work properly. I could not sit across the computer at any time" Q1.

\subsubsection{First discussion: «E-learning or face to face? Which one is better for active learning?»}

Participants' views about the first discussion session conducted as a debate are classified into three sub-themes:

- Discussion topic

- Discussion process

- Outcomes, results

According to participants, the first discussion topic is interesting, contemporary, significant, required, comprehensive and a wide range topic. Meanwhile, they appreciate debate technique.

"The topic of the discussion was almost current and needed to be discussed with the transformations in education." P8

Discussion process of the first session is described by the participants as respecting various ideas, freely indicating opposite views, sharing real-life examples, commenting on previous messages, fruitful, encouraging for participation, high-quality and well-conducted discussion.

The outcomes and results of the first discussion session was described by the participating postgraduate students as beneficial, contributing to thoughts, changing in views, gaining new knowledge, no change in views and reaching a consensus. Some participants wrote the below sentences regarding the discussion process and the results on the questionnaire.

"Everyone was respecting to each other and indicated her views freely. We reached specific results. Focus was clear and everyone indicated similar or contradictory ideas related to the focus." Q7 
"I still think that face to face learning is more effective. However, before the discussion I would certainly say face to face learning, now I can say it is possible be effective with e-learning. I can say that I started to think more openly in this topic." Q2

"I don't think my opinions changed a lot. However, I liked the views of my friends." Q3

\subsubsection{Second discussion: «Globalization and e-learning»}

Just like the first discussion session, participants' views about the second discussion session conducted as a free-flow discussion are classified into the same three sub-themes:

- Discussion topic

- Discussion process

- Outcomes, results

According to participants, the second discussion topics is interesting, comprehensive, wide range, meaningful, useful for instructors and a good selection.

"I liked it. Globalization and e-learning are nested concepts and I think we should consider in this way. Thus, such discussion topic was nice and meaningful." Q4

Participants described the second discussion session as respecting various ideas, freely indicating opposite views, supporting each other's views, respecting the borders of the topics, more abstract ideas/views, friendly environment, focusing on more than one-sub-topic, and fruitful.

Moreover, participants described the outcomes and the results of the second discussion session as contributing to thoughts, gaining new information, extending the points of view, critical thinking on the topic, and no change in views.

"Nearly all of the participants respected to others' views and handled the topic in its borders. In this aspect it was a nice discussion." Q6

"The process was efficient. We discussed the topic from various points of views and diverse views emerged. Everyone was respectful to others and freely indicated her/his views. Since we discussed the topic from various aspects, I gained new information. It was nice to see the effects of globalization. We focused on more than one sub-topics within globalization." Q7

"The views of my other participant friends were also important. I had a chance to learn new points of views." Q3

"My views about this topic has not changed as a result of the discussion, I only had a chance to grasp the topic in a wider perspective." Q2

\subsubsection{General views about the whole discussion process}

Participants' views regarding the whole discussion session in general classified into

- Moderating behaviors

- Duration of the discussions

- Shared views/ideas 
- General characteristic

- Negative aspects

Participants indicated their views regarding the motivating behaviors/tactics of the moderator. According to their views, moderating behaviors were successful, helpful for focusing, professional, carefully observing the discussions, reminding discussion rules, clarifying, helping late comers to understand the flow of the discussions.

"Moderator helped us focus on the discussion topic when the discussion was separated." Q1

“Moderator summarized all the views. This helped us follow the discussion process." Q9

Some of the participants indicated their views about the duration of the discussions. Generally speaking, the duration of the discussions were long enough.

"There was no time pressure, the required time is given to people to share their messages." Q1

Shared views and ideas were found to be helpful for gaining new insights. One of the participants stated this as follow:

"The views of other participant friends were valuable for me. I had a chance to learn new points of views from them". Q3

The general characteristic of the discussion sessions according to participants is found to be pleasant, efficient, respectful, valuable, beneficial, and effective.

"The people who participated in discussions were cultured and had large visions. I liked so much the discussion because of being respectful. ... It was pleasant." Q9

"Discussion processes were efficient. In general it was valuable and effective process." Q7.

Besides positive views, they wrote on questionnaire negative views: Having difficulty while connecting from mobile devices, low participation because of the holiday time were the highlighted views. Below statements of the participants represent these negative views:

"I commented from the smart phone but it was somehow difficult in this way." Q7

"The participation rate decreased because we could not connected from our smart phones" Q9

"I think not all of the volunteers indicated their views during the discussions. Because of the holiday time such problem might have occurred. Yet, we talk about e-learning and it is possible to reach technology from anywhere. I wish they had participated then there might have been much more discussion and active participation then." Q6

\subsubsection{Participants' suggestions}

Participants' put forwards few suggestions:

- Instead of Blackboard, using more common social media

- Adding voice into discussion process

- Conducting face-to-face discussions

- Enabling participation by using smartphones 
The statements below represent participants' suggestions:

"I think, instead on this (discussion forum tool of the Blackboard), It would be much easier and convenient if we conducted discussions on an application that we could reach anytime like WhatsApp." Q5

"I wish voice share attribute should be added, there would be more natural and heated discussions." Q1

"I would like more if we did it in face-to-face". Q5

"If the discussion platform would be an application that is reached easily from the smart phones and whenever a message is written to the group, it would give a notice, the participation might be higher." Q8

\subsection{Findings Derived From the Content Analysis of the CMC According to IAM}

Contents of the messages of the two discussions are analyzed to identify the level of social construction of knowledge and frequencies and ratios of the codings into each operations of the five phases of IAM is given on Table 2 .

Table 2. Frequency and Ratios of the Codings into Operations of Phases of IAM for the Two Discussions

\begin{tabular}{|c|c|c|c|c|c|c|c|c|c|c|c|c|c|c|}
\hline & & \multicolumn{5}{|c|}{ Phase I } & \multicolumn{3}{|c|}{ Phase II } & \multicolumn{3}{|c|}{ Phase III } & \multicolumn{2}{|c|}{ Phase IV } \\
\hline & & $\mathrm{A}$ & B & $\mathrm{C}$ & $\mathrm{D}$ & $E$ & $\mathrm{~A}$ & $\mathrm{~B}$ & $\mathrm{C}$ & $\mathrm{A}$ & $\mathrm{C}$ & $\mathrm{D}$ & $\mathrm{B}$ & $\mathrm{C}$ \\
\hline \multirow[b]{2}{*}{$\bar{a}$} & $\mathrm{f}$ & 5 & 18 & 6 & 1 & 5 & 5 & 4 & 7 & 5 & 2 & 8 & 1 & 3 \\
\hline & $\%$ & 7,14 & 25,71 & 8,57 & 1,43 & 7,14 & 7,14 & 5,71 & 10,00 & 7,14 & 2,86 & 11,43 & 1,43 & 4,29 \\
\hline \multirow{2}{*}{ ลิ } & $\mathrm{f}$ & 9 & 15 & 11 & 3 & 13 & 3 & - & 5 & - & - & - & - & - \\
\hline & $\%$ & 15,25 & 25,42 & 18,64 & 5,05 & 22,03 & 5,05 & - & 8,47 & - & - & - & - & - \\
\hline
\end{tabular}

D1: Discussion 1, D1: Discussion 2: A, B, C, D, E are the operations of the Phases of IAM.

Table 2 shows that while the debate type first discussion session reached Phase 3 and 4 in few messages, the messages of the discussion two, which was conducted as a free flow discussion, were coded into Phase 1 and Phase 2 only.

Most of the messages of the participants were coded into Phase 1: sharing/comparing of information for the whole CMC. \%25,71 percent of the codings for Discussion 1 and $\% 25.42$ percent for the Discussion 2 are coded into the Phase 1- Operation B: a statement of agreement. The statement below in one of the message is given as an example of the evidence for "statement of agreement":

"First of all I read all of your comments and I agree with all of you..." D2(4)P1(B)

Examples of other evidences in the messages for the various operations of the Phases are given below:

Phase 1 Operation A - A statement of observation or opinion:

“According to active learning I think that face-to-face is more effective." D1(5)P1(A) 
Phase 2 Operation B- Asking and answering questions to clarify the source and extent of disagreement:

"However, I would like to ask, ...while for a student one activity is enough for the others it might be limited so that is it again the case for learning preferences? Or because the information processing is different for everyone, do more activities result in negative effects?" D1(3) P2(B).

During the debate type discussion, the group decided to define the term "active learning" and coconstructed the meaning of the term in group. The given example below is taken as the evidence of one of the clarification statements for his term. Phase 3 Operation A- Negotiation or clarification of the meaning of terms:

"I agree with my friends and we should clarify what do we mean by 'active learning' firstly. We consider active learning as the process in which the learner take the responsibility of her learning, autonomous, who knows her task and conduct them, who have higher levels of awareness, has 21 st century skills...." D1(18) P3(A).

During the Discussion 1, the group selected neither e-learning nor face-to-face for active learning, they reached consensus on blended learning, and this shows negotiation/ co-construction of knowledge. Then they tested the co-constructed knowledge against their personal experience as the below message implies the advantage of blending face-to-face with e-learning by explaining a specific personal experience. Phase 4 Operation $\mathrm{C}$ - Testing against personal experience:

"This year in one of my teaching process for e-learning, the interaction with three of my students who were very silent in face-to-face classroom was very high in virtual environment. These students participated much better than the rest of the students by asking questions and producing content. I would like to state that these students planned and revised their learning process..." $\mathrm{D} 1(13) \mathrm{P} 4(\mathrm{C})$

As it is given on Table 2, Phase 3 and Phase 4 were never identified in the second discussion which was conducted as a free-flow discussion a general topic: globalization and e-learning. Table 3 shows the total frequencies and ratios of each phase of both discussions.

Table 3. Frequency and Ratios of the Codings into Phases of IAM for the Two Discussions

\begin{tabular}{lllll}
\hline Phases of the & \multicolumn{3}{c}{ Discussion I } & Discussion II \\
\cline { 2 - 5 } IAM & $\mathrm{f}$ & $\%$ & $\mathrm{f}$ & $\%$ \\
\hline Phase I & 35 & 50,00 & 51 & 86,44 \\
\hline Phase II & 16 & 22,86 & 8 & 13,56 \\
\hline Phase III & 15 & 21,43 & - & - \\
\hline Phase IV & 4 & 5,71 & - & - \\
\hline Phase V & - & - & - & - \\
\hline
\end{tabular}

According to Table 3, half of the codings were on Phase 1 for the Discussion 1 while most of the codings (86,44\%) were Phase 1 for the Discussion 2. Phase 5 is never observed in both of the discussion transcripts. These findings were compared with the previous CMC study of the author on Table 4. 
Table 4. Frequency and Ratios of the Codings into Phases of IAM for the Previous and the Current Study

\begin{tabular}{lllll}
\hline $\begin{array}{l}\text { Phases of the } \\
\text { IAM }\end{array}$ & $\begin{array}{l}\text { Previous Study* } \\
\text { All the discussions in a whole term } \\
\text { compulsory course }\end{array}$ & $\begin{array}{l}\text { Current Study } \\
\text { Two discussions together }\end{array}$ \\
\cline { 2 - 5 } & $\mathrm{f}$ & $\%$ & $\mathrm{f}$ & $\%$ \\
\hline Phase I & 206 & 80,47 & 86 & 66,67 \\
\hline Phase II & 44 & 17,19 & 24 & 18,60 \\
\hline Phase III & 5 & 1.95 & 15 & 11,63 \\
\hline Phase IV & 1 & 0.39 & 4 & 3,10 \\
\hline Phase V & - & - & - & - \\
\hline
\end{tabular}

*These findings are taken from Çardak (2016).

When the findings derived from the content analysis of the CMC transcripts according to the IAM for the previous and the current study are compared, as showed on Table 4, it is easier to see that there is a little higher levels of social construction of knowledge in the current study, though this difference is not considered as significant. While the level of social construction of knowledge in the previous study mostly remained in Phase $180,47 \%$ ), in the current study, $66,67 \%$ of the codings were into Phase 1 . The ratio of the codings into Phase 2 is very near for both of the studies. On the other hand, Phase 3 is coded only three times in the previous study, but 15 times in the current study. When Phase IV is highlighted, it is better for the current study when the total number of messages is considered for both CMC. Nevertheless, Phase V - agreement statement(s)/applications of newly constructed meaning, the highest level of social construction of knowledge, was never observed in both studies.

\section{DISCUSSION}

This study focuses on social construction of knowledge in the CMC of the postgraduate students and it is an initiative to ask what to change for the future CMC activities, comparing to previous CMC experiences, to increase the level of social construction of knowledge. The discussion is presented regarding the findings of the current and previous study of the author and other related literature. The main four differences of the current study from the previous CMC study were the voluntary participation into $\mathrm{CMC}$, familiarity of the participants with each other beforehand, moderator only role of the author and the debate type discussion. These four aspects and other issues related to the findings derived from the views of the students are discussed, results and concluding remarks are presented.

The first difference between two studies is voluntary basis for the participation. The postgraduate students of the previous study had to participate in the forum discussions in order to fulfill one of the requirement of the compulsory course and their participation in quantity was graded. Contrary to the previous case, in the current case study, there was no obligation for postgraduate students to participate in discussions, all of them were volunteers. When the findings of content analysis are compared in both studies, it is seen that the current case produced better results in terms of social construction of knowledge. On the other hand, it seems impossible to infer whether this difference is because of voluntary basis since both studies are naturalistic. In the literature, most of the research is on mandatory CMC and as Thompson and Savenye (2007) and So (2009) stated, studies on voluntary discussion sessions are less common. The literature on voluntary (non-graded) and mandatory (graded) CMC sessions is also contradictory. For instance, Johnson's (2006) states that "structured and mandated 
asynchronous discussion is associated with better cognitive outcomes than non-structured and optional discussion" (p. 51).

The voluntary vs. mandatory CMC activities should be discussed in various aspects. First of all, it should be considered within the curriculum. CMC activities within any curriculum should be congruent with the general aims of that curriculum. If this is the case, voluntary participation might be considered under the hidden curriculum rather than grading as a must component of a course. Otherwise, if the instructional aims of one of the course program of the curriculum supports conducting CMC to gain related learning outcomes, than $\mathrm{CMC}$ participation might require grading. The second aspect is affective factors that encourage or hinder the learners' voluntary and active participation into CMC activities. Voluntary participation might also be considered with gamification tactics in order to motivate learners for active participation. The other aspect for discussing voluntary participation in CMC is the informal learning need of digital society today. Informal learning seems to exceed formal learning today and informal learning is an option, not a must for the life-long learners. Individuals conduct informal learning especially on social media in a highly motivated manner. The views of the participants of the current study also points to social media as CMC system instead of forum tools of structured learning management systems. If CMC activities are transferred to social media, considered as an important medium of the informal learning today, the need for grading the participation should be considered critically, respecting the connectivist learning approach of the digital era. Experimental and multi-case studies are needed in order to understand the effect of voluntary and mandatory participation on social construction of knowledge in CMC. Thompson and Savenye (2007) recommends more studies in voluntary discussion settings in order to identify "additional drivers of learner participation", too (p.310).

The second difference between the current and the previous CMC studies of the author is the familiarity within the participants. Because the participants of the current study are in the same network of post-graduate program, they are somehow more familiar with each other than the postgraduate students of the previous study of the author. According to the comparison of two studies of the author, is it possible to infer that when the participants know each other, they negotiate the meaning and coconstruct the knowledge more easily? Answering to this question is not an easy task. One of the first initiatives to answer this question is the study of Lin and Huff in 1988. They conducted an experimental study in two groups to compare bargaining performances of the groups familiar with each other and nonfamiliar ones in CMC and face-to-face sessions, and found evidences supporting the familiarity within the subjects in CMC (Lin \& Huff, 1988). Gubta and Govindarajan (2000) states that there can be expected openness of communication when the greater familiarity and personal affinity is in a group. The study of Adams, Roch and Ayman (2005) on member familiarity and communication medium reveals that familiar participating members in CMC reach group consensus in less time and they are more satisfied, however, they may inclined to poor decision accuracy when conducting intellective tasks. Familiarity within the participants of the CMC might open them to communicate more easily and help them quest the intellectual capacity of the others. However, familiarity within a group is not the solo factors of building social presence. Possible, if the members of the group know each other before, they might trust each other, at least in means of expected behaviors. Indeed, familiarity within the group does not necessitate trust, and high level of trust within the group does not necessitate social construction of knowledge. "In CMC learning environments, some learners might do not trust each other, but they can communicate and collaborate smoothly because they are familiar with each other in terms of conversational skills, knowledge interests, expertise, etc." (Yanlin, Luyi, \& Fanglin, 2010, p. 360). Moreover, familiarity within the group might develop during the CMC if the tactics to build social presence are applied. For instance, before and during the discussions, warm-up activities are beneficial: 
The moderator may ask participants to introduce themselves and share their photographs, links to their personal profiles, let the participants communicate directly, use humors, give various roles to participants such as starter, director, summarizer, motivator.

The third difference between the current and the previous CMC studies of the author is the moderating behaviors. During the forum discussions of the previous study, the author moderated the discussions and participated as the other learners did. She was the co-participant and moderator of the $\mathrm{CMC}$ during the whole term discussions. Thought, the learner-moderator or learner-instructor interaction is important for building social presence and co-construction of knowledge, over-moderating behaviors might block learner-learner interaction as the case in the previous study. Current study produced better results when the moderator was not co-participant. She was the e-moderator who started, remind, summarized and directed to focus and closed the discussions. She waited for participants' responses before commenting during the discussion and her moderating behaviors were appreciated by the participants. Interestingly, the participants of the previous study were satisfied with the moderating behaviors, too. However co-construction of knowledge in first discussion of the current study is a little bit better, though other unestimated factors might intervene to this result. The review study of Lucas et al. (2014) on social construction of knowledge online reports that most of the discussions in reviewed studies could not go beyond Phase 1, and asserts that this might be due to the moderating behaviors. Action researches on improving moderating behaviors in CMC systems in order to enhance social construction of knowledge might be interesting.

The last difference between the structures of two CMC studies of the author is the discussion techniques used during $\mathrm{CMC}$ activities. One of the discussions of the current $\mathrm{CMC}$ was in debate format and findings of the content analysis revealed that debate type discussions produced higher levels of social construction of knowledge than the free-flow discussions of the current and the previous studies. Gunawardena et al. (1997) developed the IAM by analyzing the transcripts of debate. This might explain why the model fit better to debate type discussion of this study. On the other hand, former three structural factors and the discussion topic itself might helped participant reach Phase III and Phase IV in the debate type first discussion. Besides, most of the CMC activities are designed as sharing views about a topic, discussing a case, role-play, talks on collaborative studies of small groups, questions and answers, seminars rather than debate type of discussions. Çardak (2016) suggests conducting discussion activities on more controversial topics in order to help the participants arrive at Phase II and go beyond. Additionaly, Toulmin model of argument might be followed in CMC to reinforce participants to challenge the others during the discussions.

Apart from the discussion type and other factors, cultural attributes are also considered while interpreting the low levels of social construction of knowledge. Some cultures might not welcome dissonances/disagreements as much as stating agreements. Re-defining dissonance in the IAM regarding cultural terms is advised (Lucas et al., 2014). Cross-cultural studies might help refine the model according to specific culture, if distinct differences observed between various cultures. On the other hand, previous studies in various cultures (e.g. Hou, Chang, \& Sung, 2009; Lang, 2010; Lu \& Jeng, 2006; Paulus, 2007; Tan, Chai \& Hong, 2008; Zhao, Liang, \& Liu, 2016) report low levels of social construction of knowledge according to IAM during CMC similar to this and previous study (Çardak, 2016) conducted in Turkish culture. Indeed, today's multi-cultural societies directs instructors, teachers and curriculum experts to redesign curricula for any medium and education level according to the curricular and instructional principles of multi-cultural education. It is also a necessity for the IAM. For 
the future studies, conducting content analysis by using IAM in multi-cultural contexts might to helpful for deciding the need for differentiating dissonance in the IAM according to specific cultures.

Regrettable, Phase V, the highest level of the IAM is rarely observed in the related studies. There was no occurrence of Phase V in the current study, too. Phase V of the IAM involves summarization of agreements, application of new knowledge and participants' statements related to their learning/understanding process. With the help of authentic tasks as CMC activities, and moderator guidance to reflecting on how the participants' cognitive schema have changed during the CMC might help the occurrence of Phase V. Moreover, the participants' views on the questionnaire give some signals of Phase $\mathrm{V}$. Therefore, similar type questions of the questionnaire might be directed to participants during the $\mathrm{CMC}$ activities.

Participants' barriers to active participation might also be considered for the current study to understand other possible reasons of low level of social construction of knowledge. According to the views of the participants of the current case study, holiday time, other responsibilities and difficulty of using mobile app. of the learning management system are the main barriers for the active participation. The study of Fung (2004) reports the learners' "lack of time" as the significant barrier to participation. In fact, for the instructors, it is difficult to understand students' complaints about "lack of time", because asynchronous nature of forum discussion provides learners flexibility in time. When CMC is considered for synchronous activities, it is difficult to assign suitable date and time even for few participants, and in this case "lack of time" is understandable. Besides, individuals get used to solve their problems on smart phones at any time in anywhere. The participants of this study demands the same convenience especially for the holiday/summer days. This might be the reason why they advise using social networking sites.

\subsection{Limitations of the Study}

This study reports a CMC experience in a Turkish context and presents the level of social construction of knowledge according to IAM in addition to participants' views. The results are only valid for the case of this study and cannot be generalized to other contexts. However, the practitioners and researchers might take the case of this study as a specific example. Moreover, interaction analysis is not limited to IAM; social network analysis, qualitative content analysis of the messages, other models of discourse analysis, in-depth interviews with the participants, other quantitative results as how many messages sent by each participant, the length of the messages, time intervals between messages, etc. might give more comprehensive results for understanding the case of the current study in detail.

The results of this case study is compared with the previous study of the author in four aspects. This comparison in only based on interpretations. It means the study has not a claim about the significant effects of voluntary basis to participation, interpersonal familiarity beforehand, only moderating behavior rather than being co-participant, and debate type discussions. The author of this study, as an experienced instructor, questions her own e-moderating behaviors and structures of her CMC activities by comparing the results of her two studies. Thus, this study should be taken as an initiative to ask what to change in CMC activities for enhancing social construction of knowledge.

\subsection{Conclusion and Suggestions for the Future Research}

This naturalistic study reports the results of social construction of knowledge occurred in asynchronous forum discussions according to IAM of Gunawardena et al. (1997). The results of this 
study are compared with the previous study of the author and interpreted according to main structural differences between two CMC studies. The results of both studies show low levels of social construction of knowledge in Turkish culture, though few evidences for Phase III and IV were observed in the debate type first discussion activity of the current study. Apart from the results of content analysis of the discussion messages in the current CMC sessions, participants' views indicate satisfaction with the discussions as well as the barriers for active participation. Participants suggest using social networking sites and would like to reach the discussion environment easily from their personal learning environment on the smart phones in order to surpass the barriers. Participants' views also shows a request for using voice messages rather than only text-based messaging and conducting face-to-face discussions. This suggestions make all the practitioners think on learning preferences of each individual learner both for online and blended courses.

In conclusion, $\mathrm{CMC}$ topic is being discussed more than 30 years and the related literature still reports mostly low levels of social construction of knowledge in CMC systems. As an instructor and emoderator, the author of this study questioned her CMC activities in means of four structural aspects: voluntary/mandatory-graded, familiar/nonfamiliar, only moderator/moderator + co-participant, debate type/free-flow discussions. Although, there were not apparent differences between the results of two naturalistic case studies of the author because of these four structural differences, except for the debate type discussion, this study is an initiative to question e-educators, starting from the author herself, about what to change in CMC activities in order to reach higher levels of social contruction of knowledge. Though the comparison of the results of two CMC studies marks the debate type discussion, the possible effects of other three structural factors (voluntary participation, interpersonal familiarity, only moderating behaviors) and the context of the current study are considered while interpretting the results. This study does not imply any significant effect of discussed factors on social construction of knowledge in $\mathrm{CMC}$ but it might be taken as a starting point to conduct experimental or multicase studies on CMC systems regarding those factors. Inded, this study should be taken as a need for thinking on more radical changes and consider the term "computer-mediated discussions" in conjunction with the important concepts of the digital society: networked learning, informal learning, connectivist learning, social networking sites, personal learning environments, mobile discussions, networked discussions.

Though the discussion part provides some suggestions for the future research and for the CMC experiences of the practitioners, the following suggestions might be helpful for the future research:

- Researchers might conduct experimental mixed method design studies to compare the effects of voluntary vs. graded participation, interpersonal familiarity beforehand vs. new community, only moderator vs. moderator + co-participant roles, debate vs. other techniques of discussion on social construction of knowledge.

- Curriculum evaluation studies of online programs might help the curriculum developers estimate the value of graded forum discussions regarding the level of social construction of knowledge and make revisions on the curriculum accordingly.

- Researchers might conduct action researches to find out functional solutions to the low levels of social construction of knowledge in CMC activities.

- Phase V of the IAM might be achieved in a CMC with the help of some deliberate directions given by the moderators. Future studies might focus on developing an "e-moderating technique for networked discussions" as an online teaching technique which helps learner socially construct new knowledge. 
- Active participation in CMC activities is needed for building social presence and therefore a learning community. Learners' barriers to active participation in CMC activities might be studied in detail to understand the main barriers and produce solutions accordingly.

- Digital age learners' media preferences are important while deciding on the CMC systems. Thus, providing learners with an opportunity of selecting media and using multimedia messages in addition to text while expressing their views during CMC might be considered for the future studies.

- Social networking sites might be considered as the main medium of the CMC activities for the future case studies. Experimental studies might be conducted to identify the significant differences between the results of structured discussion forum type CMC activities and informal CMC activities on social networking sites.

\section{ACKNOWLEDGEMENT}

The author would like to thank to participating postgraduate students of the Graduate School of Educational Sciences, to independent researchers who conducted reliability studies, to three reviewers of this manuscript, and to helpful colleague who edited the language. Moreover, the author would like to thank to Dr. David Gómez, Instructional Designer at The University of New Mexico, for offering one of the discussion topic (globalization and e-learning) for the current CMC experience. 


\section{REFERENCES}

Adams, S. J., Roch, D. G., \& Ayman, R. (2005). Communication Medium and Member Familiarity: The Effects on Decision Time, Accuracy, and Satisfaction. Small Group Research, 36(3), 321-353. DOI:10.1177/1046496405275232.

Arbaugh, J. B., \& Benbunan-Fich, R. (2007). The importance of participant interaction in online environment. Decision Support Systems, 43, 853-865. DOI: 10.1016/j.dss.2006.12.013.

Çardak, Ç. S. (2016a). Increasing teacher candidates' ways of interaction and levels of learning through action research in a blended course. Computers in Human Behavior, 61, 488-506. DOI: 10.1016/j.chb.2016.03.055.

Çardak, Ç. S. (2016). Çardak, Ç. S. (2016). A Multi-Perspective Investigation into Learners' Interaction in Asynchronous Computer-Mediated Communication (CMC). Higher Education Studies, 6(2), 61-75. DOI:10.5539/hes.v6n2p61.

Fung, Y. Y. H. (2004). Collaborative online learning: interaction patterns and limiting factors. Open Learning: The Journal of Open, Distance and eLearning. 19(2), 135-149. DOI: 10.1080/0268051042000224743.

Gómez, D. R. (2018). Analyzing Social Construction of Knowledge and Social Networks in Online Discussion Forums in Spanish. Unpublished $\mathrm{PhD}$ Thesis. University of New Mexico. Retrieved from https://digitalrepository.unm.edu/oils_etds/ 52 on 2.

Gómez J. D.R., Hernández, C. M. R. (2018). Paths Toward Social Construction of Knowledge: Examining Social Networks in Online Discussion Forums. In: Meiselwitz G. (eds) Social Computing and Social Media. User Experience and Behavior. SCSM 2018. Lecture Notes in Computer Science, vol,10913.

Gunawardena, C. N., Lowe, C. A., \& Anderson, T. (1997). Analysis of a global online debate and the development of an interaction analysis model for examining social construction of knowledge in computer conferencing. J. Educational Computing Research, 17(4), 397-431. DOI:10.2190/7MQV-X9UJ-C7Q3-NRAG.

Gunawardena, C. N. \& Zittle, F. J. (1997) Social presence as a predictor of satisfaction within a computer-mediated conferencing environment, American Journal of Distance Education, 11(3), 8-26. DOI:10.1080/08923649709526970.

Gupta, K.A. and Govindarajan, V. (2000). Knowledge flows within multinational corporations. Strategic Management Journal, 24 (4), 473-496.

Heo, H., Lim, K.-Y., \& Kim, Y. (2010). Exploratory study on the patterns of online interaction and knowledge coconstruction in project-based learning. Computers \& Education 55, 1383-1392. DOI:10.1016/j.compedu.2010.06.012.

Hew, K. F., \& Cheung, W. S. (2011). Student facilitators" habits of mind and their influences on higher-level knowledge construction occurrences in online discussions: a case study. Innovations in Education and Teaching International, 48(3), 275-285. DOI:10.1080/14703297.2011.593704.

Hou, H.-T., Chang, K.-E. \& Sung, Y.-T. (2008). Analysis of Problem-Solving-Based Online Asynchronous Discussion Pattern. Educational Technology \& Society, 11 (1), 17-28.

Hou, H.-T., Chang, K.-E., \& Sung, Y.-T. (2009). Using blogs as a professional development tool for teachers : Analysis of interaction behavioral patterns. Interactive Learning Environments, 17(4), 325-340.

Johnson, G. M. (2006). Synchronous and Asynchronous Text-Based CMC in Educational Contexts: A Review of Recent Research. TechTrends, 50(4), 46-53. DOI: 10.1007/s11528-006-0046-9.

La Pointe, D. K., \& Gunawardena, C. N. (2004). Developing, testingand refining of a model to understand the relationship between peer interaction and learning outcomes in computer-mediated conferencing. Distance Education, 25(1), 83-106. DOI:10.1080/0158791042000212477. 
Lang, Q. C. (2010). Analysing high school students' participation and interaction in an asynchronous online project-based learning environment. Australasian Journal of Educational Technology, 26(3), 327-340.

Lin, J. \& Huff, S. L. (1988). Efficiency in computer mediated negotiation: The familiarity factor. ASAC Conference. Halifax, Nova Skotia. DOI: 10.20381/ruor-2263.

Lu, L. F. L., \& Jeng, I. (2006). Knowledge construction in inservice teacher online discourse. Journal of Research on Technology in Education, 39(2), 183-202. DOI:10.1080/15391523.2006.10782479.

Lucas, M., Gunawardena C., \& Moreira, A. (2014). Assessing social construction of knowledge online: A critique of the interaction analysis model. Computers in Human Behavior, 30, 524-582. DOI:10.1016/j.chb.2013.07.050.

Moore, M. G. (1989). Editorial: three types of interaction. The American Journal of Distance Education. 3(2).

Paulus, T. M. (2007). CMC modes for learning tasks at a distance. Journal of Computer-Mediated Communication, 12(4), 1322-1345. DOI: 10.1111/j.1083-6101.2007.00375.x.

Rovai, A. P., \& Barnum, K. T. (2003). On-line course effectiveness: An analysis of student interactions and perceptions of learning. Journal of Distance Education, 18(1), 57-73. Retrieved from http://www.ijede.ca/index.php/jde/article/view/121/102

Savignon, S. J. \& Roithmeier, W. (2004). Computer-mediated Communication: Texts and Strategies. CALICO Journal, 21, (2), 265-290. Retrieved from https://www.jstor.org/stable/24149395

Sher, A. (2009). Assessing the relationship of student-instructor and student-student interaction to student learning and satisfaction in web-based online learning environment. Journal of Interactive Online Learning, 8(2), 102-120. Retrieved from http://www.ncolr.org/jiol/issues/pdf/8.2.1.pdf

So, H. J. (2009). When groups decide to use asynchronous online discussions: collaborative learning and social presence under a voluntary participation structure. Journal of Computer Assisted Learning, 25, 143-160. DOI: $10.1111 / \mathrm{j} .1365-2729.2008 .00293 . x$

Su, B., Bonk, C., Magjuka, R. J., Liu, X., \& Lee, S. (2005). The importance of interaction in web-based education: Aprogram level case study of online MBA courses. Journal of Interactive Online Learning, 4(1), 1-18. Retrieved from http://www.ncolr.org/jiol/issues/pdf/4.1.1.pdf

Tan, J., Chai, S. C., \& Hong, H. Y. (2008). The analysis of small group knowledge building effort among teachers using an Interaction Analysis Model. In Computers in Education (pp. 801-808).

Thompson, E. W. \& Savenye, W. C. (2007) Adult Learner Participation in an Online Degree Program: A programlevel study of voluntary computer-mediated communication. Distance Education, 28(3), 299-312. DOI: $10.1080 / 01587910701611336$

Tu, C. H. (2000). On-line learning migration: from social learning theory to social presence theory in a CMC environment. Journal of Network and Computer Applications. 23, 27-37 DOI:10.1006/jnca.1999.0099.

Tu, C. H. (2002). The Impacts of Text-based CMC on Online Social Presence. The Journal of Interactive Online Learning. 1(2), 1-24.

Tu, C. H. \& McIsaac, M. (2002). The Relationship of Social Presence and Interaction in Online Classes. The American Journal of Distance Education, 16(3), 131-150. DOI: 10.1207/S15389286AJDE1603_2.

Yanlin, Z., Luyi, L. \&, Fanglin, Z. (2010). Social Context for Computer-supported Collaborative Learning. International Conference on Networking and Digital Society. Retrieved from https://ieeexplore.ieee.org/document/5479204

Zhao, C., Liang, Y. \& Liu, Q. (2016). Analysis of Social Network and Knowledge Construction Levels in Online Discussion. International Conference on Educational Innovation through Technology. DOI: 10.1109/EITT.2016.39, pp. 163-167. 